\title{
One flipped classroom teaching model on the course of applied optics
}

\section{Zhongjie Xu, Xiangai Cheng, Yu Ning, Weihong Hua, Hairong Zhong}

Zhongjie Xu, Xiangai Cheng, Yu Ning, Weihong Hua, Hairong Zhong, "One flipped classroom teaching model on the course of applied optics," Proc. SPIE 11143, Fifteenth Conference on Education and Training in Optics and Photonics: ETOP 2019, 1114330 (2 July 2019); doi: 10.1117/12.2523844 Photonics: ETOP 2019, 2019, Quebec City, Quebec, Canada 


\title{
One flipped classroom teaching model on the course of applied optics
}

\author{
Zhongjie $\mathrm{Xu}^{1,2,3}$, Xiangai Cheng ${ }^{1,2,3^{*}}$, Yu Ning ${ }^{1,2,3}$, Weihong Hua ${ }^{1,2,3}$ and Hairong Zhong ${ }^{1}$ \\ ${ }^{1}$ College of Advanced Interdisciplinary Studies, National University of Defense Technology, \\ Changsha, Hunan, China \\ ${ }^{2}$ Hunan Provincial Key Laboratory of High Energy Laser Technology, Changsha, Hunan, China \\ ${ }^{3}$ Hunan Provincial Collaborative Innovation Center of High Power Fiber Laser, Changsha, Hunan, \\ China
}

\begin{abstract}
Applied optics is one of the core courses for undergraduates that major in optical engineering and related fields. The course of applied optics leads to various successor courses, such as optical design, laser optics, and fiber optics, etc. It is difficult to cover all the main fields of modern optics. As an attempt, we tried to introduce the flipped classroom teaching model to improve the students' general understanding on applied optics. The course is divided into three parts: basic theory, experiments, and flipped classroom. The basic theory contains the concepts in coaxial spherical systems, prisms, apertures and aberrations, while the experiments provides deepened understandings on the basic theory. After that, the flipped classroom is introduced, including several different topics, such as telescopes, microscopes, cameras, infrared systems, projectors, fibers, etc., which refer to different fields of applied optics. The students are asked to organize contents around interested topics and give brief talks. During the last few years, several interesting topics, such as light field camera, super-resolution imaging, and 3D movie, are reviewed by students. It is found that by this teaching model, the students can get general impression of modern optics and pay more attention on their favorite topics.
\end{abstract}

Keywords: Applied Optics, Flipped Classroom

\section{INTRODUCTION}

The optical instruments play essential roles in the field of industry, social lives and scientific researches. The understanding on optical instruments have been well developed by now. The education systems on relative fields have been well established. A number of outstanding textbooks are available [1-3]. In China, the courses related on the basic knowledge of optical instruments are usually called applied optics, which is one of the core courses for undergraduates that major in optical engineering and related fields. There are several components in our course series of optical education for undergraduates, including optics, applied optics, optical design, field trip, etc. These courses aim to train different abilities in optics. The course of applied optics links the optical principles and applications. It leads to various successor courses, such as optical design, laser optics, fiber optics, and nano-optics, etc. Since there are too many relative fields in modern optics, it is difficult to cover all of them during finite classroom time of applied optics. Careful design of the whole course series is needed for higher efficiency.

We are trying to introduce the flipped classroom teaching model on the course of applied optics. The flipped classroom includes several important fields of modern optics. The main purpose of the flipped classroom is training the skills of analyzing cases of optical instruments in various fields.

This article is prepared as the following structure: in section 2 a brief introduction about the optical course series is presented. In section 3 we pay attention on the flipped classroom parts. A brief conclusion is given in section 4.

\section{THE OPTICAL COURSE SERIES}

The course series is mainly for undergraduate students. The basic course is optics, which gives basic description of wave optics and ray optics.

*E-mail: xiang_ai_cheng@126.com

Fifteenth Conference on Education and Training in Optics and Photonics: ETOP 2019, edited by

Anne-Sophie Poulin-Girard, Joseph A. Shaw, Proc. of SPIE Vol. 11143, 111433O · ( 2019

SPIE, ICO, IEEE, OSA · CCC code: 0277-786X/19/\$18 · doi: 10.1117/12.2523844 
After the students have finished the course of optics, they are asked to attend the course of applied optics, which is the major course of this series. As shown in Fig.1, there are three parts of the course: basic theory, experiments and free topics with flipped classroom. The basic theory part contains the concepts of coaxial optics, optical elements, typical optical systems and aberrations. This part aims to give a fundamental picture about the optical design and other applications. In the experimental part, there are several experiments including the observing and measuring of monochromatic aberrations, the magnification and resolution of optical imaging systems, the measuring of MTF of optical systems, etc. This part aims to give a better understanding of those basic theories, and to train basic abilities of carrying optical experiments.

After the experimental part, the students are asked to organize several free topics with the mode of flipped classroom. All topics are determined, organized, and presented by students. The attending students are asked to give brief comment and rate for the presenters. In the past years, the topics are mainly related to some important fields about optics, such as telescopes, microscopes, infrared optical systems, cameras, fiber optics, etc. With the talks and discussions about these topics, the students get an overview of the various applications of applied optics.

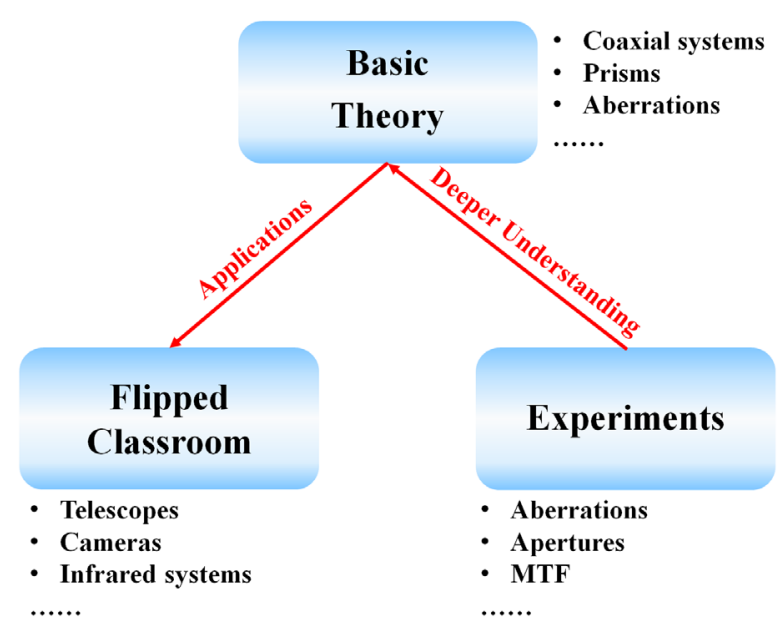

Figure 1 The course structure of applied optics.

The optical design course is rightly after the course of applied optics. The concepts of optical design are introduced. The students are asked to design several simple optical systems, such as telescopes and camera lens, with the help of modern design software. This course is an application and supplement of applied optics.

After that, the students are organized to have a field trip, in which they will get in touch with several typical optical equipment and learn to operate and assemble the equipment.

The crossover courses with other series are mainly for graduate students. One as an example is the fiber optics. The students will the concepts and measurements about the optical fibers, especially their usage in communication and laser. The skills and knowledge acquired in applied optics will be great benefit for these courses.

\section{THE FLIPPED CLASSROOM TEACHING MODEL}

The free topics part in the course of applied optics is organized with the model of flipped classroom. The students are divided into several groups. They are asked to organize contents around their interested topics and give a presentation to the whole class. After the presentation, the listeners will ask some questions and make brief discussions.

The purposes of free topics are shown in Fig.2. All topics are focused on various application scenarios. By preparing and discussion on these topics, the students are expected to improve the abilities on abstracting physical problems from practical application scenarios, choosing proper optical instruments, and the understanding of technical details of typical 
optical instruments. To achieve these purposes, the students are encouraged to compare different instruments and discuss the advantages and limitations of them, which can help the students to choose proper instruments for their applications.

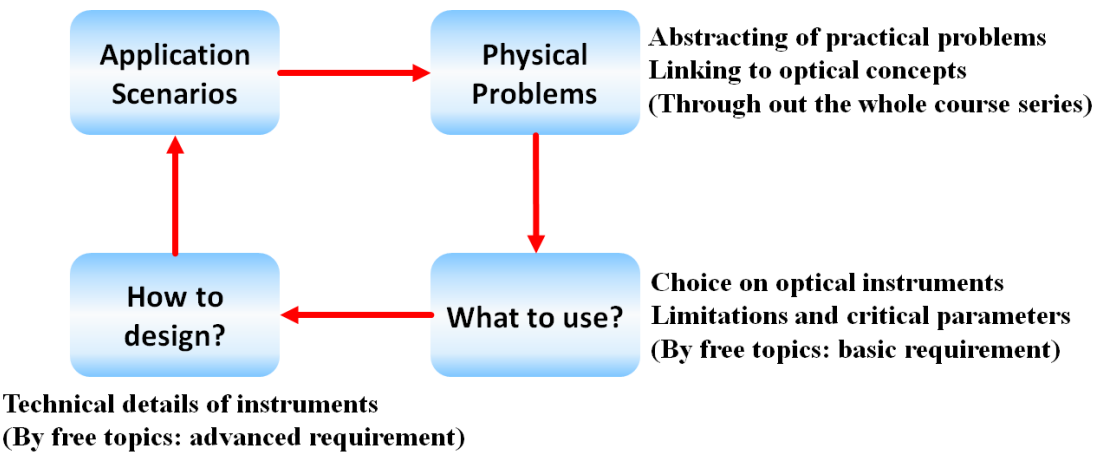

Figure 2 The purpose of free topics by flipped classroom mode.

In the past years, the favorite topics of students usually covered the fields of telescopes, microscopes, cameras, infrared systems. Some of the students also paid attention on optical fibers and AR/VR systems, etc. There were many interesting questions and discussions around the above topics. The selected topics are shown in Tab. 1

Table 1 The selected topics of flipped classroom.

\begin{tabular}{ll}
\hline \multicolumn{1}{c}{ Fields } & \multicolumn{1}{c}{ Selected Topics } \\
\hline Telescopes & $\begin{array}{l}\text { Optical telescopes and radio telescopes: similarities, differences, and applications } \\
\text { Optical theodolites: principles and history }\end{array}$ \\
\hline Microscopes & $\begin{array}{l}\text { Super-resolution imaging: STED, STORM, PALM, etc. } \\
\text { Principles and applications of phase-contrast microscope }\end{array}$ \\
\hline \multirow{2}{*}{ Infrared Optical Systems } & $\begin{array}{l}\text { Infrared night vision and low-level-light night vision: comparison and applications } \\
\text { Infrared guiding systems }\end{array}$ \\
\hline \multirow{2}{*}{ Cameras } & Discussions on 3D movie technique \\
& Light-field camera and synthetic-aperture radar (SAR) \\
\hline \hline
\end{tabular}

Take the topic of optical telescopes and radio telescopes as an example. The presenters had shown the famous optical and radio telescopes and discussed the differences including wavelengths, apertures, resolutions and working modes, etc. The questions were mainly about sensitivity and resolution, especially the different applications of single radio telescopes and radio telescope arrays, such as Five-hundred-meter Aperture Spherical Telescope (FAST) and Square Kilometer Array (SKA). A hot discussion topic this year was the Event Horizon Telescope (EHT) and the direct observation of black hole at the center of Messier 87. The possibility of optical telescope array was discussed. The students realized that the time calibration is much more difficult for shorter wavelength and optical telescope array with large distance was unavailable so far. An open question was left to the students: how would the black hole look like in visible light region?

Throughout the discussions, the students had improved their understanding on the limitations and advantages of optical telescopes and radio telescopes, and learned a lot about the details of some telescopes like Hubble, James Webber, FAST and ETH. 


\section{CONCLUSION}

In order to improve the teaching efficiency of the applied optics course, we have tried to introduce the flipped classroom teaching model to improve the students' general understanding on applied optics and its applications. The flipped classroom includes several free topics which refer to different fields of applied optics. The students are asked to organize contents around interested topics and give brief talks. The students are expected to improve the abilities on abstracting physical problems from practical application scenarios, choosing proper optical instruments, and the understanding of technical details of typical optical instruments by the preparations and discussions of free topics. During the last few years, several interesting topics, such as light field camera, super-resolution imaging, and 3D movie, are reviewed by students. It is found that by this teaching model, the students can get general impression of modern optics and pay more attention on their favorite topics.

\section{REFERENCES}

[1] Li, L. Applied Optics, Beijing Institute of Technology Press, 2006.

[2] Fischer, R. E., Optical System Design, McGraw-Hill, 2000.

[3] Smith, W. J. Modern Optical Engineering, McGraw-Hill, 2000. 